\title{
The WFCAM Transit Survey
}

B. Sipőcz ${ }^{1,3}$, G. Kovács ${ }^{2,3}$, D. Pinfield ${ }^{1}$, S. Hodgkin ${ }^{2} \&$ members of the RoPACS Initial Training Network and of the WTS consortium

${ }^{1}$ Centre for Astrophysics Research, University of Hertfordshire

[b.sipocz@herts.ac.uk]

${ }^{2}$ Institute of Astronomy, University of Cambridge

${ }^{3}$ Early Stage Researcher, RoPACS Network

\begin{abstract}
The WFCAM Transit Survey (WTS) has been obtaining data on the United Kingdom Infrared Telescope since 2007. The WTS targets about 8,000 M dwarfs over several square degrees of sky, and aims to find low-mass eclipsing binaries and planets, down to the size of the Earth, transiting M dwarf stars with periods up to a few days.
\end{abstract}

\section{Planets transiting red dwarf stars - The WFCAM Transit Survey}

Although M dwarfs form by far the largest stellar population, they are underrepresented in optical transit surveys because they are optically faint. There are currently two ways to tackle this. By concentrating on a sample of bright, nearby M-dwarfs, monitoring them one by one the MEarth team discovered the Super Earth planet GJ1214b (Charbonneau et al., 2009). We are leading a European effort using a different approach, conducting a transit survey in the near-infrared, at the peak of the $\mathrm{M}$ dwarf spectral energy distribution, using the WFCAM on UKIRT ${ }^{1}$. The WTS has been awarded 200 nights of observing time, of which $\sim 30 \%$ has been observed to date. The WTS targets 4 superfields (2 summer and 2 winter ones) in J-band, each 1.5 square degrees in size. Our total estimated sample of $\mathrm{M}$ dwarfs (Fig. 2), brighter than $\mathrm{J}=16 \mathrm{mag}$, is $\sim 8,000$ (2,000 per field) with $10 \mathrm{mmag}$ or better light curve RMS. This precision allows us to find planets down to the size from Super-Neptunes (around M0 stars) to rocky Earths (around late type M dwarf stars). For one of our summer fields we have nearly reached the proposed coverage (95\% completeness). We need to complete at least one of the other fields to have a suitably large sample of $\mathrm{M}$ dwarf stars to place meaningful constraints on the frequency and properties of $M$ dwarf planetary systems as a function of host star spectral type and planet mass. This would provide important observational constraints and helps us to better understand planet formation around low-mass stars (e.g. Laughlin et al. 2004; Ida \& Lin 2005).

${ }^{1}$ http://star.herts.ac.uk/RoPACS/

This is an Open Access article distributed under the terms of the Creative Commons Attribution-Noncommercial License 3.0, which permits unrestricted use, distribution, and reproduction in any noncommercial medium, provided the original work is properly cited. 


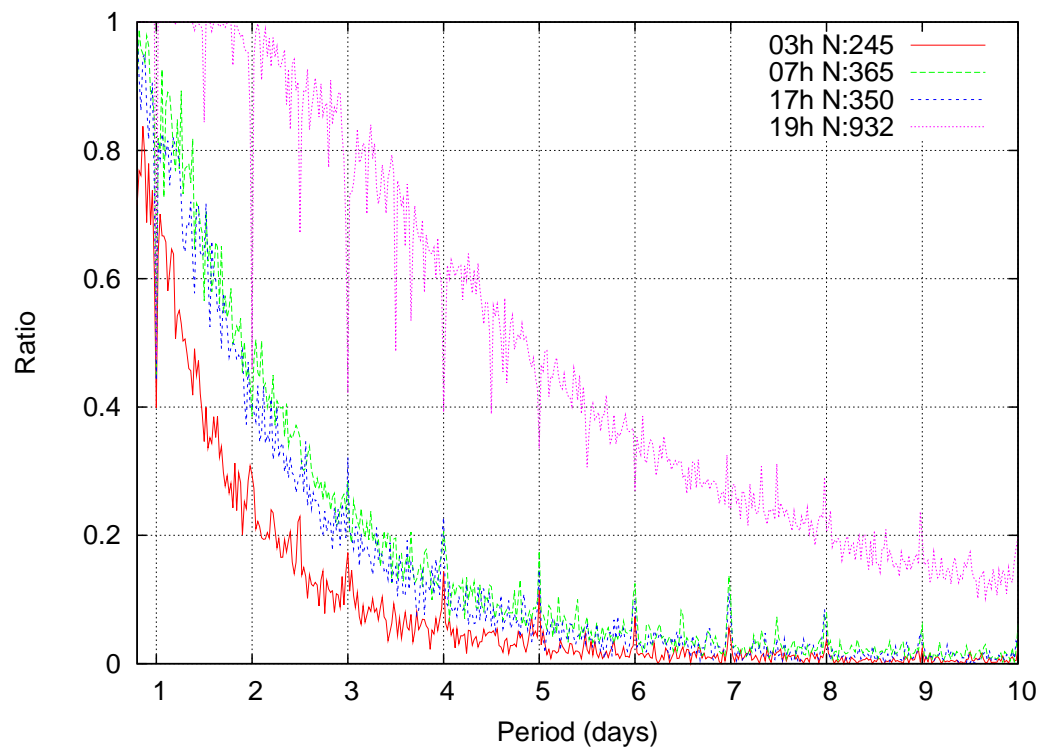

Figure 1: Recovery ratio of a 1 hour transit in the 4 fields focusing on the distribution of the observed epochs only.

\subsection{Additional science goals - Better understanding the mass radius relation}

The mass radius relation for the lowest mass stars is starting to show some surprising features with $M$ dwarfs seeming to be bigger than expected and active $M$ dwarfs even more so (Jackson et al. 2009). Studying the low-mass eclipsing binary systems (EBs), discovered in the WTS, provides useful data for this.

Our survey also provides other valuable science products, too, such as high precision light curves for studying rotation periods of low-mass stars in the field or other variability studies; multi epoch data for proper motion studies of foreground stars, e.g. cool white dwarfs and brown dwarfs; and constraints on current Galaxy models.

\section{Candidates and follow-ups}

In the 2010 summer follow-up campaign we focused on the most completed field which has significantly better coverage than the other ones. We identified 39 planet and 32 low-mass EB candidates in this field.

Follow-up light curves were obtained for 10 planet candidates using the INT and LT telescopes. Low resolution spectra, suitable for spectral typing, were obtained for all of the planet candidates using the WHT and CAHA 3.5m telescopes. For 5 of them, we have $\mathrm{km} / \mathrm{s}$ precision RV curves taken with the WHT. One candidate was sent to the HET to obtain high precision high signal-to-noise spectra, suitable for detecting the RV variation of the star due to its planetary companion. We have also followed up a selection of low-mass EBs. Nine of them got follow-up light curves using the INT and LT telescopes and for 4 of them we have RV curves obtained by the WHT. Their publication is in preparation. 
Detection and Dynamics of Transiting Exoplanets
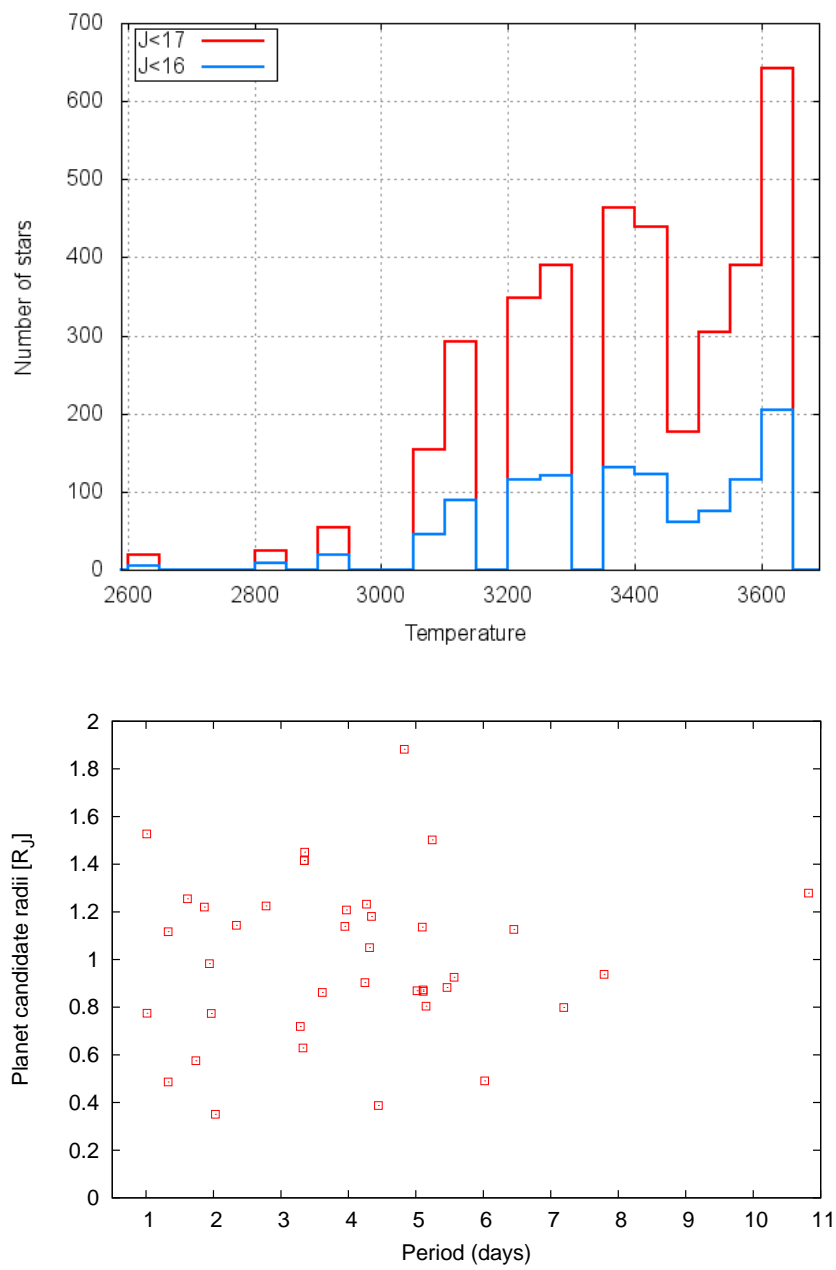

Figure 2: Upper panel: Number of $M$ dwarfs as a function of $T_{\text {eff }}$. $T_{\text {eff }}$ estimation is based on SED fitting using 9 photometric bands from combined Sloan and UKIRT data. Blue line is for stars $J<16$ magnitude and red is for $J<17$. Lower panel: Estimated radius of the WTS planet candidates.

Acknowledgements. BS and GK are supported by RoPACS, a Marie Curie Initial Training Network funded by the European Commission's Seventh Framework Programme.

\section{References}

Charbonneau D. et al. 2009, Nature 462, 891

Ida S. \& Lin D. 2005, ApJ626, 1045

Laughlin G. et al. 2004, ApJ612, 73 